\section{Methods of identification models of soil humidity}

\section{ZH Aliyev*}

Institute of Soil Science and Agrochemistry of the National Academy of Sciences of Azerbaijan, Azerbaijan

For the operational forecasting of the dynamics of moisture reserves, it is reduced to the prediction of precipitation and total evaporation (E). The remaining elements of the balance either do not change over time, are either known or are defined as functions of $\mathrm{P}$ and $\mathrm{E}$.

The plant's need for water E (evapotranspiration) is determined on the basis of the bioclimatic method in the modification of B.V. Danilchenko (2) by the formula:

$\mathrm{Eu}=\mathrm{E} \mathrm{K} \mathrm{b} \mathrm{K} \mathrm{M}$

Where $\mathrm{E}$ is the total evaporation;

To $b$ is the biological coefficient of culture;

KM - microclimatic coefficient. Ivanov

In this case, evaporation is determined by the formula of

$\mathrm{E}=\mathrm{ki} \mathrm{dt}(\mathrm{v})$

where $\mathrm{Ki}$ is the temperature coefficient characterizing the energy part of the evaporation;

d - elasticity vapor;

$f(v)$ is the wind function.

When calculating the volatility per day, the temperature coefficient is determined by the formula:

$\mathrm{Kt}=61 \times 10^{-1}(25+\mathrm{t})^{2} \mathrm{ix}^{-1}$

where $t$ is the air temperature;

Ia is the vapor pressure in mb.

Deficiency of vapor pressure is defined as

$\mathrm{D}=(1-0.1 \mathrm{r})$

where $r$ is the relative humidity in percent.

Wind function can be determined by

$f(v)=0.64(1+0.19 v r)$
More Information

*Address for Correspondence: ZH Aliyev, Professor, Institute of Soil Science and Agrochemistry of the National Academy of Sciences of Azerbaijan, Azerbaijan, Tel: +994504242130; Email: zakirakademik@mail.ru

Submitted: 09 March 2020

Approved: 02 June 2020

Published: 03 June 2020

How to cite this article: Aliyev ZH. Methods of identification models of soil humidity. Ann Civil Environ Eng. 2020; 4: 034-037.

DOI: 10.29328/journal.acee.1001024

Copyright: @ 2020 Aliyev ZH. This is an open access article distributed under the Creative Commons Attribution License, which permits unrestricted use, distribution, and reproduction in any medium, provided the original work is properly cited.

(2) Check for updates

( ) Open Access

here vr is the veteran's speed 2 meters above the surface of the earth, in m / s;

$\mathrm{vr}=0.1 \mathrm{vf} ;$

vf is the wind speed at the height of the wind vane.

One solution is to use rolling operational forecasts.

Operational irrigation plans are calculated once or twice a week, when forecasting moisture reserves in every 1 st period 10 days before. In the next 1 st period, actual changes in stocks over the past day are calculated.

Given the new initial wetting, a forecast is left for the next 10 days. For two equal variables $\mathrm{P}$ and $\mathrm{E}$, only total evaporation is predicted promptly taking into account the expected variables, and the amount of precipitation is always taken equal to zero.

This approach is associated with the organization of irrigation: the prediction of rainfall mobilizes in preparation for irrigation, in the case of the prediction of heavy rainfall is easy to stop.

Operational forecasting of total evaporation E, Starting field moisture is defined as

(a)

$\mathrm{W}=\mathrm{hjb}$

(a) 
Where - $\mathrm{h}$ - active soil layer;

$\mathrm{b}$ - average soil moisture, to the mass of dry soil; $\mathrm{F}$

$\mathrm{j}$ is the average soil density for the layer.

Moisture reserve at the end of the day

$\mathrm{W} \tau=\mathrm{W} \tau-1+(\mathrm{P} \tau-\Delta \mathrm{P} \tau)-\mathrm{W} \tau \mathrm{q}+\mathrm{m} \tau-\mathrm{E} \tau$

Here $W \tau-1$ is the moisture supply at the end of the previous and the beginning of new days in mm;

$\mathrm{m} \tau$ - net irrigation rate (which is entered into the calculation if irrigation was carried out on the day) in mm;

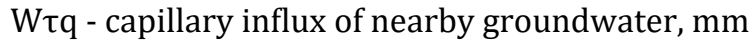

$\Delta \mathrm{P} \tau$ - precipitation loss on surface runoff and filtration, $\mathrm{mm}$

$\mathrm{P} \tau$ - the amount of precipitation, in $\mathrm{mm}$

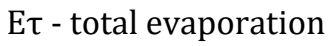

$\mathrm{E} \tau=\varphi \tau \mathrm{E} \tau+(1+\varphi \tau) \mathrm{Etp} ; \varphi \tau \leq 1:$

Daily value of total evaporation:

where - $\varphi \tau$ is the characteristic of the degree of field coverage by plants, determined from the relation:

an $\tau$ is the degree of coverage of the plant field observed per day;

an $\mathrm{kp}$ is the critical value;

E 1 - evaporation from part of the field by vegetation, $\mathrm{mm}$;

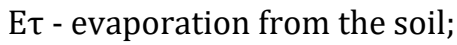

E $\tau$ - calculated through bioclimatic coefficient

(kp) / (kp)

E $\tau$ for an $\tau<$ an $E \tau$ for an $\tau>$ an

Wherein

$e^{-0^{\prime} 3(\tau-1)}$

En $\tau=E n t$ and $E \tau=K \sigma(E u) \tau$ Eu $\tau$

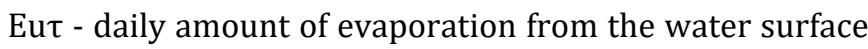
of the GTI-3000 evaporator

$\kappa \sigma(\mathrm{Eu}) \tau$ - biological coefficient of total evaporation, changing in phases, according to a curve specific for each culture

For the day of moistening, $\tau=0$. Evaporation is calculated in various ways: for the first and second half of the day.

The soil moisture model known in the works of G.K. Aslanov (3) for decade data on the mountainous regions of Azerbaijan is known:
- for the rise phase of daily water consumption, the humidity model has the form:

$$
\mathrm{W}=0.697 \mathrm{X}-1.788 \mathrm{Y}-1.412 \mathrm{Z}+48.428
$$

- for the decline phase, daily water consumption:

$\mathrm{W}=0.079 \mathrm{X}-6.568 \mathrm{Y}+177.089$

The boundary between the phases of rise and fall was the end of the second decade of July.

Here $\mathrm{W}$ is the moisture reserve at the end of the decade, $\mathrm{mm}$;

$X$ - moisture supply at the beginning of the decade and the amount of precipitation per decade;

$\mathrm{Z}$ - average moisture deficit per decade mm;

0

$\mathrm{Y}$ - average decade temperature in $\mathrm{C}$

In [2], regression equations are presented that express the statistical relationship between the GTK parameter

R

SCC $=$

$0,1 \sum \mathrm{t}$

Where - $\sum \mathrm{t}$ is the sum of air temperatures;

$\mathrm{P}$ is the amount of precipitation in $\mathrm{mm}$.

A significant factor, impacting on water consumption crops are their biological characteristics. It is believed that each biological species of a plant is characterized by its own defined rhythm of development and the corresponding regime of water consumption.

The influence of biological characteristics of crops on the dynamics of water consumption is taken into account using biological coefficients of total evaporation of $\mathrm{Ki}$, determined by the formula:

$$
E=E \sigma \sum d
$$

Where $\mathrm{E}$ is the water consumption for the entire growing season;

$\sum \mathrm{d}$ is the sum of moisture deficits for that period,

$\mathrm{K} \sigma$ is the coefficient of the biological curve, determined by dividing the gross water flow rate for the interphase period by the sum of water moisture deficits.

The biological curve coefficient is influenced by many factors, in the area, weather conditions. Under different climatic conditions, their values can vary 1.5-2 times, and therefore, their refinement is required. In general, the soil moisture model has the form. 


$$
{ }^{d W_{q}}
$$

dt

Here $\mathrm{W}$ is the soil moisture.

It is advisable to replace such differential equations in identification problem such as above for ontogenesis with the difference ones obtained by integration. The quality of soil characteristics affecting its moisture content should be considered: Here,

$\mathrm{T}$ is the type of soil

$\tau_{R-}$ in mountainous areas the time of solar radiation

a - irrigation intensity,

$\mathrm{T}_{\mathrm{n}}$ - time after the last watering,

$S$ is the intensity of solar radiation,

$\hat{G}$ - planting density of the plant,

$\mathrm{T}_{\mathrm{n}-}$ soil temperature at depths of $0.2 \mathrm{~m}, 0.4 \mathrm{~m}, 0.6 \mathrm{~m}, 1.2 \mathrm{~m}$. $Q_{s}$ - infiltration

$\mathrm{A}$ is the thickness of the active soil layer,

\begin{tabular}{|l|l|l|}
\hline Culture & $\begin{array}{l}\text { Regression Equation } \\
\text { The southern slope of the Greater } \\
\text { Caucasus }\end{array}$ & $\begin{array}{l}\text { Multiple } \\
\text { regression coefficient }\end{array}$ \\
\hline Tea & 0.43 SCC +2.62 & 0.74 \\
\hline Corn & 0.12 SCC +3.2 & 0.69 \\
\hline Winter wheat & 0.28 SCC +4.77 & 0.43 \\
\hline & Southeast slope Greater Caucasus & \\
\hline Vineyards & 0.028 SCC $\div 0.31$ & 0.870 \\
\hline Two year old alfalfa & 0.016 GTK $\div 0.505$ & 0.58 \\
\hline Winter wheat & $0.004 \div 0.583$ & 0.32 \\
\hline
\end{tabular}

$\mathrm{T}_{\mathrm{qs}}$ - soil surface temperature,

$\mathrm{V}_{\mathrm{TR}}$ - transpiration volume or sowing volume,

$\mathrm{R}$ is the precipitation intensity vector,

$\mathrm{T}_{\text {about }}$ - a vector of time of rainfall.

Identification was carried out using a step-by-step regression program using the inclusion method to select variables.

These indicators are significant when a large area with different soil characteristics in different areas is considered. However, for small areas, the task of adapting the soil moisture model is not necessary, and a one-time "Start" identification of its parameters is sufficient. It should, however, consider that when "small" amounts of land in the mountainous terrain, with heavy lateral inflow adaptation humidity models can be necessary. The estimation of the parameters of the soil moisture equation is also impossible in form (21) and requires a transition to form (25).

$t_{1}$

$$
\Delta \mathrm{W}_{\mathrm{q}}=\int \mu\left(\mathrm{W}_{\mathrm{q}}, \mathrm{S}\right) \mathrm{dt}
$$

$t_{2}$

In [1] B.G. Aliev a general model of soil moisture is presented in the form:

(H) $-0.31\left(\tau_{1}\right)$

$\mathrm{W}_{\mathrm{q}}=\mathrm{W}_{1}$ e e

(h)

where, $\mathrm{W}_{1}$ - evaporation in the first day after watering,

$\tau$ is the day ordinal after wetting.

The calculated ratios for the first and second half of the day are significantly different. However, this does not take into account the dependence on wind speed and temperature. In the work [3] of G. K. Aslanov the evaporation equation is given, having the form:

$\mathrm{E}_{0}=0,0018\left(25+\mathrm{t}^{2}\right)(100-\mathrm{a})$

Here $t$ is the average temperature

a is the average value of humidity

$\mathrm{bE}_{0 .}$

$\mathrm{W}_{\mathrm{H}}\left[1-\cdots+-\mathrm{y}_{\mathrm{n}}\right.$

$2 y$

$\mathrm{W}_{\mathrm{k}}=$

$\mathrm{bE}_{0}$

$1+$

$2 y$

To estimate the moisture reserves in the soil layer one meter thick at the beginning and end of each growing season in (3) the equation is shifted:

where $\mathrm{b}$ is the dimensionless empirical coefficient,

$y$ - food moisture reserves of the soil with less moisture

$\mathrm{k}$ - filed root layer from the lower layers of soil:

$\mathrm{J}$ - infiltration beyond the root layer, aeration zone

soil

$y_{n}-$ runoff. 
Another model is also proposed to characterize the condition of natural moisture in the mountainous territories of Azerbaijan:

$$
(1-\mu) X+W_{\mu}-W_{k}
$$

$\mathrm{a}=$

\section{E}

where, $-\mu$ is the drain coefficient,

$\mathrm{X}$ is the amount of precipitation,

W - moisture reserves in the calculated soil layer at the beginning and end of the estimated time period,

$\mathrm{E}$ is evaporation.

Identification of the model soil moisture was carried out based on the data for the harvested area.

\section{References}

1. Aliev BH, Aliev IN, Aliev ZH. Technique and technology of lowintensity irrigation in the conditions of the mountainous region of Azerbaijan. Publishing House "Elm" Baku 1999: 220.

2. Aliev BH. Problems of desertification in Azerbaijan and ways to solve it. Publishing House "Ziya-Nurlan. Baku. 2005: 670.

3. Aliyev BH, Aliyev IN. Problems of erosion in Azerbaijan and ways to solve it. Publishing House ZIYA-CPI “Nurlan”, Baku. 2000; 122.

4. Benet HH. Fundamentals of soil protection. M.L. 1958.

5. Kovda VA. Problems of combating desertification, salinization of irrigated soils. M., Kolos, 1984.

6. Izyumov AN. Erosion in the mountainous regions of Azerbaijan and the fight against it. Baku, 1954

7. Ibragimov AA. Some issues of accounting for flushing and mapping of eroded zones. "Complex erosion control measures in action" Volume II methods of accounting and evaluation of erosion and hydrological indicators. Voroshilovgrad 1985

8. Synchronous pulse sprinkling. Publishing House "Selhozizdat". M Moscow. 1984; 212 